\title{
Advanced maternal age
}

Kate F Walker MRCOG, PhD Clinical Assistant Professor of Obstetrics and Gynaecology, Division of Child Health, Obstetrics and Gynaecology, School of Medicine, University of Nottingham, Queen's Medical Centre, Nottingham University Hospitals NHS Trust, Nottingham, UK. Conflicts of interest: none declared.

Jim G Thornton FRCOG, MD Professor of Obstetrics and Gynaecology, Division of Child Health, Obstetrics and Gynaecology, School of Medicine, University of Nottingham, Maternity Department, Nottingham City Hospital, Nottingham University Hospitals NHS Trust, Nottingham, UK. Conflicts of interest: none declared

\section{Abstract}

The average age of women at childbirth in industrialised nations has been increasing steadily for approximately 30 years. Women aged 35 years or over have an increased risk of gestational hypertensive disease, gestational diabetes, placenta praevia, placental abruption, perinatal death, preterm labour, fetal macrosomia and fetal growth restriction. Unsurprisingly, rates of obstetric intervention are higher among older women. Of particular concern is the increased risk of antepartum stillbirth at term in women of advanced maternal age. In all maternal age groups, the risk of stillbirth is higher among nulliparous women than among multiparous women. Women of advanced maternal age ( $>40$ years) should be given low dose aspirin (in the presence of an additional risk factor for pre-eclampsia) and offered serial ultrasounds for fetal growth and wellbeing; given the increased risk of antepartum stillbirth, induction of labour from 39 weeks' gestation should be discussed with the woman.

Key words: advanced maternal age; pregnancy complications; antepartum stillbirth 


\section{Epidemiology}

The average age at childbirth in the UK is increasing, and more women are giving birth over the age of 35 years. In 1996, 12\% of live births were to women over the age of 35 years, by 2006 that figure had risen to $20 \%$ and by 2017 it had further risen to $23 \%$. The Office of National Statistics estimated that in $2006,4.6 \%$ of live births were to nulliparous women aged between 35 and 39 years of age and $0.9 \%$ were to women over the age of 40 , by $2017,6.4 \%$ of livebirths were to nulliparous women between 35 and 39 years of age and $1.2 \%$ were to women 40 years and older.

The reasons for delaying childbirth are complex and multi-factorial and it is important to remember that while some women may make a conscious choice to delay childbearing, it may be the unwelcome sequelae of subfertility for others.

\section{Antenatal effects}

\section{Maternal}

Women over 35 years are at higher risk of many pregnancy complications. Is this simply because women over 35 years are also more likely to have pre-existing medical conditions than their younger counterparts, pre-disposing them to developing pregnancy complications? In a large prospective cohort study of over 1.5 million deliveries during a 15 year period in Sweden, Jacobsson et al examined the relationship between advanced maternal age (AMA) and adverse perinatal and obstetric outcomes, and what contribution pre-existing maternal medical conditions had on the occurrence of adverse outcomes in women over 40 years of age. The study observed that women over 40 years of age were at increased risk of pregnancy 
complications and this risk persisted when the data was adjusted for pre-existing maternal disease.

A retrospective observational study of over 150,000 deliveries in Canada considered the relationship between maternal age and adverse outcomes and adjusted for potential confounders, including pre-existing maternal medical disease. A smaller retrospective study of 630 women in Italy examined the relationship between maternal age and pregnancy complications but did not adjust for pre-existing maternal disease. Both of these studies found that pre-existing maternal disease was more common with advancing maternal age.

Women over 40 years have a higher risk of developing gestational diabetes than women aged 20-30 years of age (OR 3.4).

There is some controversy over whether women of AMA are at increased risk of hypertensive disorders in pregnancy. In the previously mentioned Swedish cohort study, women over 40 years of age were found to have a higher risk of developing pregnancy-induced hypertension and severe pre-eclampsia, although they were at a slightly lower risk of developing mild preeclampsia. The increased risk of severe pre-eclampsia and pre-eclampsia as a whole were increased when the data was adjusted for pre-existing maternal disease and smoking. There was an increased risk of hypertensive disorders (including pregnancy-induced hypertension and pre-eclampsia) in the Canadian study, after adjusting for pre-existing maternal disease in women over 35 years of age (adjusted rate ratio 2.32) and in women over 40 years of age (adjusted rate ratio 3.55). No relationship between maternal age and hypertensive disorders was found in the Italian study, perhaps owing to the smaller sample size. 
Women over 40 years of age are at higher risk of placenta praevia (OR 4.6 - 5.8) and placental abruption (adjusted rate ratio 1.8).

A national, population-based cohort study using the UK Obstetric Surveillance System (UKOSS) published in 2016, reported on the outcomes for women giving birth aged $\geq 48$ years. They found that when the data was adjusted for confounders, women were more at risk of gestational diabetes (AOR 4.81; 95\% Cl 1.93 - 12); caesarean section (AOR 2.78; 95\% Cl 1.44 - 5.37) and admission to an intensive care unit (AOR 33.53; 95\% $\mathrm{Cl} 2.73-412$ ).

\section{Fetal}

The pregnancies of women over 35 years are at higher risk of perinatal death; this encompasses antepartum and intrapartum stillbirth and early neonatal death.

The Centre for Maternal and Child Enquiries (CMACE) report that perinatal deaths from 2441 completed weeks' gestation affect $0.8 \%$ of all pregnancies to women over the age of 35 years, and $1 \%$ of all pregnancies to women over the age of 40 years. The distribution of maternal age among the mothers having perinatal deaths is significantly higher than the general maternity population. Mothers having stillbirths and neonatal deaths are more likely to be older (40+ years).

Women of advanced maternal age are at increased risk of preterm labour, both at less than 32 weeks' gestation (ARR 1.36 for women over 35 and ARR 2.41 for women over 40) and at less than 37 weeks' gestation (ARR 1.61 for women over 35 and ARR 1.8 for women over 40). Women of AMA are at increased risk of bearing a low birth weight infant $(<2500 \mathrm{~g})$. Conversely women of AMA are also at increased risk of bearing a macrosomic (>3999g) infant and the potential complications associated with macrosomia. 
Interestingly, it has been reported that women over 35 years of age typically believe that their age puts their infant at increased risk.

\section{Late pregnancy/intrapartum complications}

\section{Obstetric intervention}

The overall Caesarean rate for nulliparous women less than 35, 35-39 and $>40$ years old is $21 \%, 38 \%$ and $50 \%$ respectively. The caesarean rate for nulliparous women in labour (both spontaneous and induced) at term, excluding breech presentation, is $22.8 \%$ among women 35-39 years of age and $27.4 \%$ among women aged 40 years or older (unpublished data, Scottish maternities 2004-2008). In nulliparous women, the relationship between maternal age and delivery by emergency Caesarean is linear, which suggests a biological effect of advancing maternal age on labour performance, rather than simply obstetrician or maternal preference. Smith et al investigated this possible biological effect of advancing maternal age on myometrial contractility further. They took uterine biopsies from the upper edge of the lower segment uterine incision from 62 women at the time of their elective caesarean section between 38 and 40 weeks' gestation. The myometrial tissue was dissected into longitudinal strips and examined in vitro for its inherent contractility. They found a non-significant trend towards a reduction in frequency of spontaneous contractions in myometrial fibres with advancing maternal age. Overall, they found a significant reduction in spontaneous contractile activity with maternal age and this effect persisted when the results were adjusted for advancing gestational age and number of previous vaginal deliveries. The risk of operative vaginal delivery also increased linearly with maternal age in nulliparous women. The rates of 
operative vaginal delivery in women $<35,35-39$ and $>40$ years were $23.5 \%, 36.9 \%$ and $43 \%$ respectively.

When the length of labour was examined in over 120,000 women who had a singleton, term, live, cephalic birth and who reached $10 \mathrm{~cm}$ dilatation for both nulliparous and multiparous women the duration of the first stage of labour decreased with advancing maternal age, and the duration of the second stage in nulliparous women increased with advancing maternal age.

\section{Perinatal death}

Perinatal death refers to the death of a fetus or a newborn in the perinatal period that commences at 24 completed weeks' gestation and ends before seven completed days after birth. It therefore includes stillbirth (antepartum and intrapartum) and early neonatal death.

Stillbirth accounts for $67 \%$ of all perinatal deaths; excluding intrapartum causes, antepartum stillbirth accounts for $61 \%$ of all perinatal deaths and is by far the commonest cause of perinatal death at term. Antepartum stillbirth is particularly important in women over 40 years of age because they are less likely to have future pregnancies. Female fecundity (the ability to produce offspring) declines with age, with monthly fecundity gradually decreasing from the mean age of 30 years onward. If the mean rate of fecundity per cycle for women aged $20-30$ years is scaled as 1 , for a woman aged 33 years of age the relative rate is 0.75 . In addition to the reduction in natural fertility with increasing age, there is a significant decrease in the chance of having a healthy child after the age of 30 . Beyond 30 years of age the chance of a 'healthy baby' decreases by 3.5\% each year. Assisted reproductive technologies cannot entirely counteract nature, the live birth rate for IVF/ICSI among women less than 39 years is 
$27.3 \%$ whereas for women aged between $40-42$ years this rate is $11.1 \%$, decreasing to $4 \%$ in women over 44 years of age.

Maternal age 35 years and over is associated with a $65 \%$ increase in the odds of stillbirth and accounts for over 4000 stillbirths in high income countries each year. The increasing average age at first childbirth may be contributing to the static rates of antepartum stillbirth in the UK over the last 20 years. One possible strategy to consider is an earlier induction of labour for women of advanced maternal age.

\section{Antepartum stillbirth}

\section{Choosing the correct denominator}

Traditionally, the risk of perinatal death at term was expressed as the perinatal mortality rate (the number of all perinatal deaths divided by the number of births in a given week). However, when considering antepartum stillbirth, Smith et al suggest that the population of babies at risk at any given gestational week are not only the babies born that week, but all babies yet to be delivered. Therefore it is important to consider the cumulative risk of stillbirth at any gestational age (the number of antepartum stillbirths at a given gestational week divided by the number of ongoing pregnancies at that gestational week). Although the perinatal mortality rate is lowest at 41 weeks, the gestational age associated with the lowest cumulative risk of perinatal death is 38 weeks. 


\section{The risk of antepartum stillbirth at term}

The overall cumulative risk of antepartum stillbirth throughout gestation (from 20 to 41 completed weeks) for women of all ages is 6.5 per 1000 pregnancies. The cumulative risks of stillbirth for women younger than 35 years, 35-39 years and older than 40 years old, were 6.2, 7.9 , and 12.8 respectively.

The largest increase in cumulative risk of stillbirth for women over 35 years of age starts at 39 weeks and peaks at 41 weeks. Women over 40 years old have a similar stillbirth risk at 39 weeks as women who are between 25 and 29 years old at 41 weeks, and once they pass 40 weeks' gestation their risk of stillbirth exceeds that of all women $<40$ years old at term. To quantify that risk, the risk of stillbirth between 37-41 weeks for women aged 35-39 years old is 1 in 382 ongoing pregnancies (RR 1.32), and for women 40 years or older 1 in 267 ongoing pregnancies (RR 1.88). If this is expressed as a risk per 1000 ongoing pregnancies, 2.6 in 1000 for women 35-39, and 3.7 in 1000 for women aged 40+, then comparisons with the routine practice of offering induction of labour postdates can be drawn, due to a 2-3 in 1000 risk of stillbirth for women of all ages.

It is a widely accepted practice in the UK to offer induction of labour for a pregnancy that continues beyond 41 weeks, termed a postdates pregnancy. This is because the rate of stillbirth increases six-fold from 0.35 per 1000 ongoing pregnancies at 37 weeks to 2.12 per 1000 ongoing pregnancies at 43 weeks. Neonatal (up to 28 days) and post neonatal (from 28 days - 1 year of age) mortality falls significantly with advancing gestation up until 41 weeks, when it plateaus and then increases with prolonged pregnancy. As such the overall risk of pregnancy loss (stillbirth plus death occurring up to the age of 1 year) increases 8 fold between 37 weeks and 43 weeks, justifying induction of labour at 41 weeks. 


\section{Antepartum fetal monitoring}

An alternative solution to induction of labour at term for women of advanced maternal age at increased risk of late antepartum stillbirth, is to perform antepartum fetal surveillance. Could this alone identify those babies at increased risk of stillbirth, rendering induction of labour unnecessary?

In a modelling comparison of 3 different strategies: a policy of usual care (no antepartum testing or induction of labour before 41 completed weeks), testing from 37 weeks with induction of labour for a positive test, and no testing with induction of labour at 41 weeks, there was a calculation of the number of antenatal tests, inductions of labour and caesarean sections that would arise and the number of fetal deaths that would be averted if each strategy was adopted. The type of antepartum testing used was not specified. In the group with 'normal management', between 37 and 41 weeks, women aged over 35 years were modelled to experience 5.2 unexplained fetal deaths per 1000 pregnancies. In the group with antepartum testing from 37 weeks with induction of labour for a positive result, 3.9 fetal deaths per 1000 would be avoided, but this would require 863 antepartum tests, 71 inductions and 14 additional caesarean deliveries per fetal death averted. A strategy of induction at 41 weeks would avert 0.9 fetal deaths per 1000 pregnancies and require 469 inductions and 219 additional caesareans per fetal death averted. The conclusion was that antepartum testing should be initiated over induction at 41 weeks.

A retrospective cohort study of over 15,000 women who delivered at a single hospital in Los Angeles investigated surrogate markers of uteroplacental insufficiency in women 35 years of age and older, such as stillbirth, birth weight less than the $10^{\text {th }}$ percentile, fetal distress, caesarean section for fetal distress, oligohydramnios, meconium passage and Apgar at 5 
minutes of less than 7 , to see whether maternal age over 35 years was an independent risk factor for uteroplacental insufficiency. Of all the markers, two (intrapartum fetal distress and caesarean delivery for fetal distress) were significantly more common in the older cohort, but all other markers were the same in each cohort. The increased risk of stillbirth in women of advanced maternal age could not be entirely explained by uteroplacental insufficiency, and there was deemed to be insufficient evidence to recommend routine antepartum fetal monitoring for all women over 35 years of age.

Umbilical and fetal Doppler ultrasound is used widely as an antepartum surveillance tool to assess placental function and confirm fetal wellbeing. Its use in pregnant women deemed to be 'high' and 'low' risk as a screening tool has been evaluated in Cochrane Reviews. 'Highrisk' women are broadly defined as women who have either a medical condition such as diabetes, or a pregnancy-related condition such as antepartum haemorrhage which are associated with an increased perinatal morbidity or mortality. In 'high-risk' women the use of Doppler ultrasound was associated with a statistically significant reduction in perinatal mortality (RR 0.71) and caesarean delivery (RR 0.90). There was a non-statistically significant reduction in stillbirths, neonatal deaths and low Apgar scores. The authors concluded that the use of Doppler ultrasound in high-risk women improved perinatal outcomes, without adversely effecting maternal outcomes. A Cochrane review of the use of Doppler ultrasound in 'low-risk' women found no conclusive evidence that Doppler ultrasound confers fetal or maternal benefits. If the small increased risk of antepartum stillbirth in women of advanced maternal age were largely due to placental insufficiency, then it is possible that Doppler ultrasound could provide a useful screening tool for detecting those women most at risk of stillbirth. 


\section{Aetiology of stillbirth in women of advanced maternal age}

Are particular causes of antepartum stillbirth more common in women of advanced maternal age?

Traditional methods of classifying the cause of stillbirth (for example the Wigglesworth approach) leave two thirds of cases 'unexplained'. In 2008, CMACE, who report on perinatal deaths in the United Kingdom, introduced a new classification system which reduced the proportion of 'unexplained' stillbirths to $23 \%$. They found the most common causes were haemorrhage (13\%), intrauterine growth restriction (IUGR) (10\%) and placental (9\%). They found that women aged 35 and over were less likely to experience a stillbirth due to IUGR.

Twenty percent of stillbirths are associated with intra-uterine growth restriction. Of those stillbirths where IUGR was a primary cause or contributing factor, $29 \%$ of cases occurred in women aged between $25-29$ years old versus only $5 \%$ of cases occurring in women aged $40+$

years. Indeed, in an examination of whether the relationship between maternal age and unexplained stillbirth was due to an increased risk of IUGR in women of advanced maternal age, the stillborn infants of women aged over 35 years were found to be more likely to be normally grown than growth restricted. This would suggest that antenatal screening with Doppler ultrasound for placental dysfunction in women of advanced maternal age may be ineffective.

A study of 76 cases of unexplained antepartum stillbirth found that the chance of experiencing an unexplained stillbirth without IUGR increased by $16 \%$ for each year increase in maternal age. 
A study of 715 cases of stillbirth examined whether particular causes of stillbirth were more common in women aged 35 and over. The authors found that stillbirths to women aged over 35 were more likely to be due to infection, (odds ratio, OR 2.8), 'unusual causes' such as cord pathology (OR 2.0) or be 'unexplained' (OR 2.2). Another study of 76 cases of unexplained antepartum stillbirth found that the chance of experiencing an unexplained stillbirth without IUGR increased by $16 \%$ for each year increase in maternal age.

We performed an observational study using anonymised national data on 2850 cases of antepartum stillbirth in 2009 in the UK to breakdown the main causes of antepartum stillbirth by maternal age, and identify if specific causes are more common in older mothers. We found that stillbirths in women aged 35 years and over are more likely to be due to major congenital anomalies (Relative Risk Ratio (RRR) 2.0, 95\% Cl 1.3-3.0), mechanical causes (RRR 1.6, 95\% Cl 1.0-2.6), maternal disorders (RRR 2.1, 95\% Cl 1.2-3.6) or associated obstetric factors (RRR 2.1, $95 \% \mathrm{Cl} 1.1-3.9)$ than women less than 35 . Women aged 35 years and over have a statistically significant increased risk of stillbirth due to major congenital anomalies (OR relative to live birth 1.6, 95\% $\mathrm{Cl} 1.3-1.9)$ and maternal disorders (OR 1.7, 95\% $\mathrm{Cl} 1.2-2.4$ ) than younger women. Women aged 35 years and over were $30 \%$ more likely to experience a term stillbirth than women $<35$ years (OR $1.3,95 \% \mathrm{Cl} 1.1-1.5)$. Stillbirth due to congenital anomaly was statistically significantly more likely in women $\geq 35$ years.

Advanced maternal age is a significant risk factor for antepartum stillbirth particularly at term. Attention should be given to stillbirth due to mechanical causes, maternal disorders and associated obstetric factors in such women.

Lean et al examined the placentas of a cohort of women of maternal age $\geq 35$ years (96 women) and a control group of younger women (aged 20-30 years, 47 women), all with 
uncomplicated pregnancies. All women in the study delivered liveborn term infants weighing $>2.5 \mathrm{~kg}$. They found that the placentas of women of advanced maternal age displayed increased syncytial nuclear aggregates and decreased proliferation, and had increased amino acid transporter activity, all histological findings associated with stillbirths complicated by fetal growth restriction. They also found a difference in placental hormones in women of AMA typically associated with placental dysfunction and stillbirth associated with fetal growth restriction, lower PAPP-A and soluble fms-like tyrosine kinase-1 (sFlt). This study suggests that placental dysfunction may be more common in women of advanced maternal age and could be a contributor to their observed increased risk of stillbirth.

\section{Parity and stillbirth}

Are parous women of advanced maternal age at lower risk of antepartum stillbirth than nulliparous women?

A large retrospective cohort study of over 600,000 women in Sweden found that nulliparous women were at a higher risk of stillbirth than multiparous women.

In a large retrospective study of over 5 million deliveries across the USA between 2001 and 2002, the relationship between parity, advanced maternal age and stillbirth was examined. While nulliparous and multiparous women had a similar increase in the risk of stillbirth with advancing maternal age, nulliparous women had a higher risk of stillbirth than multiparous women for all maternal age groups. Thus the risk of stillbirth at 37 weeks per 1000 ongoing pregnancies in women aged $<35,35-39$ and $40+$ was $3.72,6.41$ and 8.65 in nulliparous women and 1.29, 1.99 and 3.29 in multiparous women. 
The contribution that parity plays to the risk of antepartum stillbirth in women of AMA has been extensively investigated. It is fair to conclude that nulliparous women have a higher risk of stillbirth than multiparous women for all maternal age groups.

\section{Neonatal morbidity}

Much of the focus of the literature has been on the relationship between advanced maternal age and perinatal death. Valent et al (2016) found an increased risk of a composite of neonatal morbidity associated with advanced maternal age; 35-39 years ARR 1.11, 40-44 years 1.21 , and $45-49$ years 1.34 when the data was adjusted for factors known to increase the risk of neonatal morbidity.

Women's experience of pregnancy at an advanced maternal age

An important study by Southby et al has examined women's experience of pregnancy at advanced maternal age. They performed one to one in-depth interviews with nulliparous women aged between 35 and 44 years old. The central category identified by the authors was that women had a 'now or never' mentality and that concerns about their future opportunities to have more babies was the most important category affecting their experience of pregnancy. We highly recommend this article to the reader.

\section{Management of advanced maternal age and pregnancy}

\section{Reducing the risk of hypertensive disorders in pregnancy}

Maternal age 40 years of older constitutes a moderate risk factor for the development of preeclampsia. In the presence of one additional risk factor, NICE recommend that $75 \mathrm{mg}$ of aspirin should be prescribed from 12 weeks until delivery. Given the increased risk of hypertensive disorders as with all pregnant women, care should be taken to offer advice on 
achieving a healthy lifestyle and the symptoms of pre-eclampsia and meticulous attention should be given to blood pressure and urinalysis for proteinuria at each antenatal visit.

\section{Surveillance for fetal growth restriction}

Maternal age 40 years or older constitutes a major risk factor for having a small-forgestational-age neonate and therefore serial ultrasounds for growth and umbilical artery Doppler from 26-28 weeks should be offered according to RCOG guidance.

\section{Consideration of induction of labour from 39 weeks' gestation}

We conducted a randomised, controlled trial involving nulliparous women who were 35 years of age or older. Women were randomly assigned to labour induction between 39 weeks 0 days and 39 weeks 6 days of gestation or to expectant management (i.e., waiting until the spontaneous onset of labour or until the development of a medical problem that mandated induction). The primary outcome was caesarean delivery. The trial was not designed or powered to assess the effects of labour induction on stillbirth. The results showed that induction of labour at 39 weeks of gestation, as compared with expectant management, had no significant effect on the rate of caesarean section. Moreover, maternal and neonatal outcomes and women's experience of childbirth did not differ significantly between the groups assigned to these strategies. We performed an economic evaluation alongside the trial and found that the intervention was associated with a mean cost saving of $£ 263$ and a small additional gain in QALYs (though this was not statistically significant), even without considering any possible QALY gains from stillbirth prevention. Consideration should be given to offering women of advanced maternal age induction of labour from 39 weeks' gestation. 


\section{Conclusion}

The average age of women at childbirth in industrialized nations has been increasing steadily for approximately 30 years. Women aged 35 years or over have an increased risk of hypertensive disease, gestational diabetes, placenta praevia, placental abruption, perinatal death, preterm labour, fetal macrosomia and fetal growth restriction. Unsurprisingly, rates of obstetrical intervention are higher among older women. Of particular concern is the increased risk of antepartum stillbirth at term in women of advanced maternal age. Women of advanced maternal age should be given to low dose aspirin (in the presence of an additional risk factor for pre-eclampsia), offered serial ultrasounds for fetal growth and wellbeing and induction of labour from 39 weeks gestation given the increased risk of antepartum stillbirth should be discussed with the woman.

\section{Further reading}

Jacobsson B, Ladfors L, Milsom I. Advanced maternal age and adverse perinatal outcome. Obstet Gynecol. 2004; 104(4):727-33.

Reddy UM, Ko CW, Willinger M. Maternal age and the risk of stillbirth throughout pregnancy in the United States. Am J Obstet Gynecol. 2006;195(3):764-70.

Smith GCS, Cordeaux Y, White IR, Pasupathy D, Missfelder-Lobos H, Pell JP, et al. The effect of delaying childbirth on primary cesarean section rates. Plos Med. 2008; 5(7): 1123-32.

Walker KF, Bradshaw L, Bugg GJ, Thornton JG. Causes of antepartum stillbirth in women of advanced maternal age. Eur J Obstet Gynecol Reprod Biol. 2016; 197:86-90. 
Walker KF, Bugg GJ, Macpherson M, McCormick C, Grace N, Wildsmith C, et al. Randomized Trial of Labor Induction in Women 35 Years of Age or Older. N Engl J Med. 2016; 374(9): 81322.

\section{Practice points}

- Maternal age 40 years of older constitutes a moderate risk factor for the development of pre-eclampsia.

- Maternal age 40 years or older should be offered serial ultrasounds for growth and umbilical artery Doppler from 26-28 weeks.

- Consideration should be given to offering women of advanced maternal age induction of labour from 39 weeks' gestation. 